\title{
Epidural blood patch for an eight-months' history of post-dural puncture headache: case report
}

\begin{abstract}
Long-term complications due to spinal anaesthesia though rare have devastating implications as far as quality of life is concerned. One such complication is postdural puncture headache ((PDPH). The objective of this report is to review the diagnosis, pathophysiology of PDPH and give a brief description of the therapeutic options available.

A 31-year-old healthy female was referred to our chronic pain clinic with complaints of headache for eight months following spinal anesthesia. She reported that simple analgesics and supine position provided partial pain relief. The headaches without these measures were incapacitating. An epidural blood patch was performed with complete resolution of the headache. At two and four weeks reviews she reported no headache and was discharged from the pain clinic without any complaints.

This case demonstrated that the epidural blood patch is one of the armaments at the disposal of the anesthetists for effective treatment of chronic post-dural puncture headache.
\end{abstract}

Keywords: anesthesia, spinal, blood patch, post-dural puncture headache, epidural
Volume 10 Issue 3 - 2018

\section{Jesse M Mumba}

Specialist Anaesthesiologist, Ondangwa Private Hospital, Namibia

Correspondence: Jesse M Mumba, M.D (Tver), MMed Anaesthesia (UCT), FCA(SA), Specialist Anaesthesiologist, Ondangwa Private Hospital, Namibia, +264 8I 754 9600, Email jesse@jesmuira.com

Received: May 12, 2018 | Published: June 20, 2018

\section{Introduction}

Post-Dural Puncture Headache (PDPH) is a well-known and expected complication after perforation of the dura mater with spinal needles. Its pathophysiology is not completely understood. ${ }^{1-4}$ The epidural blood patch (EBP) is the most effective of the therapeutic strategies and has been recommended both as a diagnostic and treatment modality for persistent headache after dural puncture. ${ }^{5}$

\section{Case report}

We report a case of a 31-year-old female patient referred to our chronic pain clinic with eight months' history of severe chronic headaches. Patient history reviewed no comorbidities that could explain the signs and symptoms she presented with. The headache was predominantly frontal, non-pulsatile, and worsened when the patient was standing, sitting or leaning forward with some relief reported in the supine position. She denied the presence of nausea, tinnitus, hearing symptoms, neck or dorsal rigidity. No neurological deficits were elicited during physical examination. CT brain was ordered but patient could not afford. Fundoscopy was normal. Further probe into the history, characteristics of the headache and what alleviated it reviewed that the headaches started on the same day she had hystorotomy under spinal anaesthesia for severe preeclampsia at 27 weeks' gestation. She took simple analgesics with mild relief over the eight months that followed. Chronic post dural puncture headache was suspected as the cause of the chronic headaches. The spinal anaesthesia for hystorotomy was done with a Spinocan 22G cutting needle at $\mathrm{L} 4 / 5$. The number of attempts were not recorded.

Epidural blood patch (EBP) was performed with a $18 \mathrm{G}$ Tuohy needle with full sterile precautions. Epidural spaced was identified with loss of resistance to saline at L3/4 space in the lateral decubitus position. Twenty milliliters of autologous blood was drawn from antecubital vein. Upon injection of $15 \mathrm{mls}$, patient reported "heaviness" in the lower back and no further blood was injected. Patient was then put in supine position for 3 hours. The $5 \mathrm{mls}$ of blood that was left in the syringe was kept at body temperature until a clot formed at 7 minutes. She was discharged 6 hours later from the hospital without headache. At two and four weeks review she she reported no headaches since EBP and was discharged from the chronic pain clinic.

\section{Discussion}

The International Headache Society defines PDPH headache occurring within 5 days of a lumbar puncture, caused by cerebrospinal fluid (CSF) leakage through the dural puncture. It is usually accompanied by neck stiffness and/or subjective hearing symptoms. It remits spontaneously within 2 weeks, or after sealing of the leak with autologous epidural lumbar patch. ${ }^{6}$ Typically, the headaches are described as affecting the occipital or frontal regions. In severe cases, posterior neck pains, hearing and visual symptom, nausea and vomiting accompany the symptomatology of PDHP. ${ }^{7,8}$

The pathogenesis of PDPH is not fully understood. Literature review supports two main hypotheses. The first is that of loss of buoyance effect of the cerebral spinal fluid (CSF) on the brain leading to traction on structures. ${ }^{1}$ This is further explained by worsening of headache in orthostatic position. An average $450-600 \mathrm{ml}$ of CSF is produced and absorbed per day in an adult i.e. $0.28-0.42 \mathrm{ml} / \mathrm{min}$. At any given time, the total volume of CSF in the adult is $\sim 150 \mathrm{ml}$, of which $125 \mathrm{ml}$ distributes in cranial and spinal subarachnoid spaces and $25 \mathrm{ml}$ in the ventricles. This explains why small leaks from dural fistula can result in significant changes in CSF pressures. ${ }^{9}$ Normal CSF pressure in the horizontal position varies from 5 to $15 \mathrm{~cm} \mathrm{H}_{2} \mathrm{O}$; however, after neuraxial puncture this pressure can be lower than $4 \mathrm{~cm}$ 
$\mathrm{H}_{2} \mathrm{O} .{ }^{10-12}$ Although this sounds like a plausible pathogenesis of PDPH, there are radiological studies that do not support traction of brain structures as a cause. ${ }^{13}$

The second hypothesis can be looked at in the light of the MonroeKelly doctrine. This doctrine states that the skull is a rigid vault containing CSF, blood and brain matter. A reduction in any of the three constituents causes a compensatory increase in the remaining two and vice versa to maintain the intracranial pressure. The constant loss of CSF via the dura defect causes intracranial hypotension. The compensatory veno-dilatation due to activation of adenosine receptors is thought to be a potential cause of PDPH. ${ }^{14}$ Fibroblast proliferation at the site of dura perforation leads to collagen formation which forms a permanent seal closing off the orifice. This process begins approximately after 48 hours, and it continues for seven days. Many authors attribute PDPH to failure of collagen formation leading to formation of chronic CSF fistula with consequent leak of CSF. Vandam et'al report that $72 \%$ of PDPH resolve within 7 days while follow up at 6 months shows $87 \%$ resolution. ${ }^{15}$

Several factors impact the incidence of PDPH. Among the notable ones are; age, type and size of needles and number of attempts at passage through the dura. ${ }^{16,17}$ Children younger than 13 years and adult older than 50 years have less incidences of PDPH largely due to reduced CSF pressure. ${ }^{18,19}$ However, gender does not seem to be a factor if the age differences other co-founding factors such as pregnancy are corrected for. ${ }^{1,5,20}$ Other risk factors for PDPH include low body mass index (BMI), taller height of patient, sitting position during dural puncture, bevel orientation, non-smoking, lower systolic blood pressures and perioperative use of dexamethasone. ${ }^{5}$ The Figure 1. Below illustrates the incidence of PDPH depending on the type of needles used.

\begin{tabular}{lcc|}
\hline Atraucan & $5 \%(26 \mathrm{G})$ & Combination Quincke-pencil point bevel \\
\hline Eldor & $0 \%(26 \mathrm{G})$ & Double hole pencil point \\
\hline Gertie Marx & $0 \%-4 \%(24 \mathrm{G})$ & Single port pencil point \\
\hline Quincke & $2.7 \%-19 \%(29 \mathrm{G}-25 \mathrm{G})$ & Cutting edge \\
\hline Spinocan & $39 \%(22 \mathrm{G})$ & Single port pencil point \\
\hline Sprotte & $1.5 \%-2.8 \%(24 \mathrm{G})$ & Single port pencil point \\
\hline Whitacre & $0.37 \%-39 \%(22 \mathrm{G}-27 \mathrm{G})$ & \\
\hline
\end{tabular}

Figure I Incidence of PDPH after different spinal needles. Used with Permission. ${ }^{5}$

Two therapeutic strategies are mainly employed in the management of PDPH, conservative and conventional invasive management. Conservative measures include psychological support, adequate hydration of the patient, use of abdominal binders and administration of oral simple analgesics and methylxanthines. In patients presenting with nausea and vomiting, anti-emetics are cardinal in alleviating the symptoms. Paracetamol, non-steroidal and caffeine are commonly prescribed. Gamma aminobutyric acid (GABA) analog such as gabapentin and pregabalin have been reported to be superior to caffeine/paracetamol in reduction of pain, nausea and vomiting. ${ }^{21}$ The efficacy of conservative management strategies have little evidence but most clinicians will use them in the hope that closure of CSF fistula would take before invasive management can be instituted.

Conventional invasive management involves placement of subarachnoid catheters in case of accidental dura puncture (ADP) as preventative measure and epidural blood patch for already developed PDPH. EBP follows the same technique as the epidural placement with the injection of $10-20 \mathrm{ml}$ of autologous blood. The performance of EBP has the same contraindications that normally apply to epidural anesthesia.

The EBP has dual benefits. It can be used both as diagnosis and treatment modality once other causes of headaches have been excluded..$^{1-4,22}$ In cases like ours where there is absence of radiological studies to rule out headaches due to intracranial pathology, simple bedside test can be performed. These are based on the understanding of transduced pressure. Application of constant abdominal pressure with one hand and the other hand placed on the lumber region for 2030 minutes while observing the intensity of the pain before and after removal of abdominal pressure is one such test. The characteristic pain of PDPH disappears or decreases upon compression and returns after release of the abdominal pressure. Another is digitally applying pressure on the carotid artery which worsens the headache while pressure on the internal jugular vein improves it. Headaches due to other causes do not alter intensity during these maneuvers. ${ }^{5,23}$

Standard EBP procedure was performed on our patient eight month after the spinal anaesthesia. Patient reported no headaches at 2 hours post EBP and the lower back "heaviness" reported after injections of $15 \mathrm{cc}$ of blood resolved. She was discharged from the hospital 6 hours later. Patient was discharged from the chronic pain clinic at 4 weeks having complained of no headache at the second review.

Headaches that do not resolve following neuraxial anaesthesia even after expected resolution period should alert the clinician to 
the possibility of chronic PDPH. After excluding organic causes of chronic headaches and available physician Anaesthetist who is well vested with the siting of epidurals, EBP can be used both as a diagnostic and therapeutic procedure as in the case presented here if there is history of dural puncture.

\section{Acknowledgements}

None.

\section{Conflict of interest}

The author declares no conflict of interest

\section{References}

1. Turnbull DK, Shepherd DB. Post-dural puncture headache: pathogenesis, prevention and treatment. Br J Anaesth. 2003;91(5):718-729.

2. Klepstad P. Relief of postural post dural puncture headache by an epidural blood patch 12 months after dural puncture. Acta Anaesthesiol Scand. 1999;4(9):964-966.

3. MacArthur C, Lewis M, Knox EG. Accidental dural puncture in obstetric patients and long term symptoms. BMJ. 1993;306:883-885.

4. Ferre JP, Gentil ME. Seven months delay for epidural blood patch in post-dural puncture headache. Eur J Anesthesiol. 1999;16:257-258.

5. Wang F. Post Dural Puncture Headache-We Can Prevent It. In: Racz GB, Noe CEBT-P, editors. Rijeka: In Tech; 2014.

6. Classification and diagnostic criteria for headache disorders, cranial neuralgias, and facial pain. Headache Classification Committee of the International Headache Society. Diagnostic criteria 7.2.1. Post-dural puncture headache. Cephalalgia. 2013:88.

7. Gaiser R. Postdural puncture headache. Curr Opin Anaesthesiol. 2006;19:249-253

8. Yentis SM, Haire K. Epidural blood patch for atypical headache following obstetrical epidural analgesia. Anaesthesia. 1997;52(1):6264

9. Wadud R, Laiq N, Qureshi FA, et al. The frequency of postdural puncture headache in different age groups. Journal of the College of Physicians and Surgeons-Pakistan. 2006;16(6):389-392.

10. Sakka L, Coll G, Chazal J. Anatomy and physiology of cerebrospinal fluid. European Annals of Otorhinolaryngology Head and Neck Diseases. 2011;128(6):309-316.
11. Kuczkowski KM. Post-dural puncture headache in pregnant women: What have we learned? Revista Colombiana de Anestesiología. 2006; 34(4):267-272.

12. Schwartz KM, Luetmer $\mathrm{PH}$, Hunt $\mathrm{CH}$. Position-related variability of CSF opening pressure measurements. AJNR American Journal of Neuroradiology. 2013;34(4):904-907.

13. Grant R, Condon B, Hart I, et al. Changes in intracranial CSF volume after lumbar puncture and their relationship to post-LP headache. $J$ Neurol Neurosurg Psych. 1991;54(5):440-442.

14. Fearon W. Post-lumbar puncture headache. P\&S Medical Review. 1993.

15. Vandam LD, Dripps RD. Long-term follow-up of patients who received 10,098 spinal anesthetics: failure to discover major neurological sequelae. JAMA. 1954;156:1486-1491

16. Schmittner MD, Terboven T, Dluzak M, et al. High incidence of postdural puncture headache in patients with spinal saddle block induced with Quincke needles for anorectal surgery: a randomised clinical trial The International Journal of Colorectal Disease. 2010;25(6):775-781.

17. Schmittner MD, Urban N, Janke A, et al. Influence of the pre-operative time in upright sitting position and the needle type on the incidence of post-dural puncture headache (PDPH) in patients receiving a spinal saddle block for anorectal surgery. The International Journal of Colorectal Disease. 2011;26(1):97-102.

18. Tobias JD. Postdural puncture headache in children-etiology and treatment. Clinical Pediatrics. 1994;33(2):110-113.

19. Tourtellotte WW, Henderson WG, Tucker RP, et al. A randomized, double-blind clinical trial comparing the 22 versus 26 gauge needle in production of the post-lumbar puncture syndrome in normal individuals. Headache. 1972;12(2):73-78.

20. Bernards CM. Epidural and spinal anesthesia. In: Barash PG, Cullen BF, Stoelting RK, editors. Clinical Anesthesia. Nova York: Lippincott Williams \& Wilkins; 2006. p. 691-617.

21. Kokki H, Turunen M, Heikkinen M, et al. High success rate and low incidence of headache and neurological symptoms with two spinal needle designs in children. Acta Anaesthesiologica Scandinavica. 2005;49(9):1367-1372.

22. Wilton NC, Globerson H, Rosayro AM. Epidural blood patch for postdural puncture headache: its never too late. Anesthesia and Analgesia. 1986;65(8):895-896.

23. Cooper G. Epidural blood patch. Eur J Anaesthesiol. 1999;16:211-215. 